\title{
Long-term survival and tumor 5-FU sensitivity in patients with stage IV colorectal cancer and peritoneal dissemination
}

\author{
SAYURI HASEGAWA ${ }^{1}$, MASAYA MUKAI ${ }^{1}$, SHINKICHI SATO ${ }^{2}$, HIROMI NINOMIYA ${ }^{2}$, KANAKO WAKUI $^{2}$, \\ NOBUKAZU KOMATSU ${ }^{2}$, TAKAYUKI TAJIMA ${ }^{4}$, HISAO NAKASAKI ${ }^{3}$ and HIROYASU MAKUUCHI ${ }^{4}$ \\ ${ }^{1}$ Department of Surgery, Tokai University Hachioji Hospital, Ishikawa-cho 1838, Hachioji, Tokyo 192-0032; \\ Departments of ${ }^{2}$ Pathology, and ${ }^{3}$ Surgery, Tokai University Oiso Hospital, Gakkyou 21-1, Oiso, Kanagawa 259-0198; \\ ${ }^{4}$ Department of Surgery, Tokai University School of Medicine, Bohseidai, Isehara, Kanagawa 259-1193, Japan
}

Received December 19, 2005; Accepted February 2, 2006

\begin{abstract}
Among 125 patients with peritoneal dissemination (P1-3) of colorectal cancer, including those with other synchronous metastases, the 5-year overall survival (OS) rate was $13.3 \%$ for P1 patients $(\mathrm{n}=30), 12.8 \%$ for $\mathrm{P} 2$ patients $(\mathrm{n}=39)$, and $1.8 \%$ for $\mathrm{P} 3$ patients $(\mathrm{n}=56)(\mathrm{P} 1$ vs. $\mathrm{P} 2$, $\mathrm{p}=$ N.S.; $\mathrm{P} 2$ vs. $\mathrm{P} 3, \mathrm{p}=0.02$; $\mathrm{P} 1$ vs. $\mathrm{P} 3, \mathrm{p}=0.001$ ), while the median survival time (MST) was $12.0,14.1$, and 3.1 months, respectively. The 5-year OS rates for patients who had peritoneal dissemination without other metastases were $17.6 \%(\mathrm{n}=17), 12.5 \%(\mathrm{n}=19)$, and $3.4 \%(\mathrm{n}=28)(\mathrm{P} 1$ vs. $\mathrm{P} 2$, $\mathrm{p}=\mathrm{N} . \mathrm{S}$.; $\mathrm{P} 2$ vs. P3, $\mathrm{p}=\mathrm{N} . S$.; P1 vs. $\mathrm{P} 3, \mathrm{p}=0.039$ ), while the MST was 25.1, 15.1, and 12.5 months, respectively. In the P3 short survival group (SSG; $\mathrm{n}=13$ ), TS expression was high in $7.7 \%(1 / 13)$ and low in $92.3 \%(12 / 13)$ of tumors, while DPD expression was high in 38.5\% (5/13) and low in $61.5 \%$ (8/13) of tumors. In the P3 long survival group (LSG; $\mathrm{n}=15)$, the corresponding values were $80.0 \%(12 / 15), 20.0 \%$ (3/15), $33.3 \%(5 / 15)$, and $66.7 \%(10 / 15)$. High TS and low DPD expression was found in only 7.7\% (1/13) of the SSG tumors vs. $46.7 \%(7 / 15)$ of the LSG tumors $(\mathrm{p}=0.028)$. These results suggest that the prognosis of stage IV colorectal cancer with $\mathrm{P} 3$ peritoneal dissemination is extremely poor. In addition, patients fitting the SSG criteria are unlikely
\end{abstract}

Correspondence to: Dr Sayuri Hasegawa, Tokai University Hachioji Hospital, Department of Surgery, Ishikawa-cho 1838, Hachioji, Tokyo 192-0032, Japan

E-mail: mukoyama.sayuri@hachioji-hosp.tokai.ac.jp

Abbreviations: 5-FU, 5-fluorouracil; LV, leucovorin; TS, thymidilate synthase; DPD, dihydropyrimidine dehydrogenase; OS, overall survival; MST, median survival time; SSG, short survival group; LSG, long survival group; CPT-11, irinotecan; L-OHP, oxaliplatin; NS, not significant

Key words: chemosensitivity, colorectal cancer, 5-fluorouracil, peritoneal dissemination, dihydropyrimidine dehydrogenase, thymidilate synthase to respond to treatment with 5-FU+LV, and may need combination chemotherapy using CPT- 11 and/or L-OHP.

\section{Introduction}

The incidence of various cancers, such as colorectal, lung and breast cancer, has been increasing in Japan as the lifestyle and diet of Japanese people become more westernized. A review of deaths due to malignant tumors in Japan showed that colorectal cancer ranked 4th in men after lung, gastric and liver cancer, and ranked 2nd in women after gastric cancer (1). It is known that $30-40 \%$ of patients with stage III primary colorectal cancer complicated by lymph node metastasis develop hematogenous metastasis/recurrence in organs such as the liver or lungs. In addition to postoperative adjuvant chemotherapy, various interventional treatments for these patients have been reported, including the resection of metastases and hepatic artery infusion therapy (2-4). The percentage of patients who develop peritoneal dissemination is lower in the case of colorectal cancer compared with gastric cancer (5). Although occasional cases of long postoperative survival have been reported, there have been few investigations into the long-term prognosis of colorectal cancer after palliative resection (6-8). Peritoneal dissemination can be explained by the following two mechanisms: i) intraperitoneal dispersion of cancer cells via the lymphatics; and ii) direct spread of tumor cells from a site of serosal invasion leading to the growth of new lesions after peritoneal implantation (9-11). It has been reported that approximately $30-40 \%$ of gastric cancer patients who have lymph node metastases develop recurrence with peritoneal involvement. Thus, peritoneal dissemination may also be explained by the direct spread of tumor cells from the greater omentum to the parietal peritoneum when lymphatic obstruction due to local lymph node metastases causes retrograde flow of lymph into the greater omentum (12).

In Japan, systemic administration of 5-fluorouracil (5-FU) and leucovorin (LV) has been the standard first-line postoperative chemotherapy for stage III to IV colorectal cancer complicated by lymph node metastasis (13-15). It has been reported that the response rate to 5 -FU monotherapy is approximately $20-25 \%$, while the response rate increases to $\geq 30 \%$ when LV is added as a modulator of 5-FU $(13,14)$. 


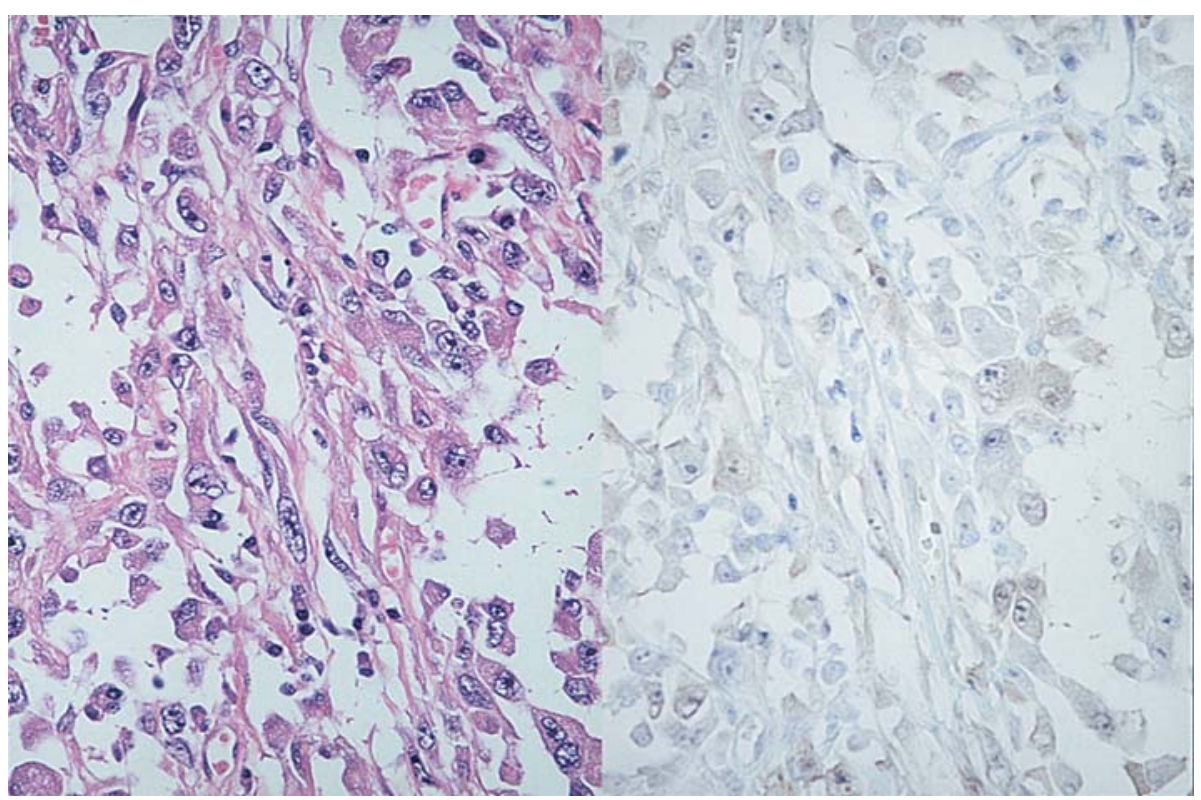

Figure 1. Immunohistochemical staining for thymidilate synthase (+++, high expression;) in the primary tumor of a stage IV colorectal cancer patient with peritoneal dissemination from the long survival group (left, H\&E stain; right, TS stain). Original magnification, x200.

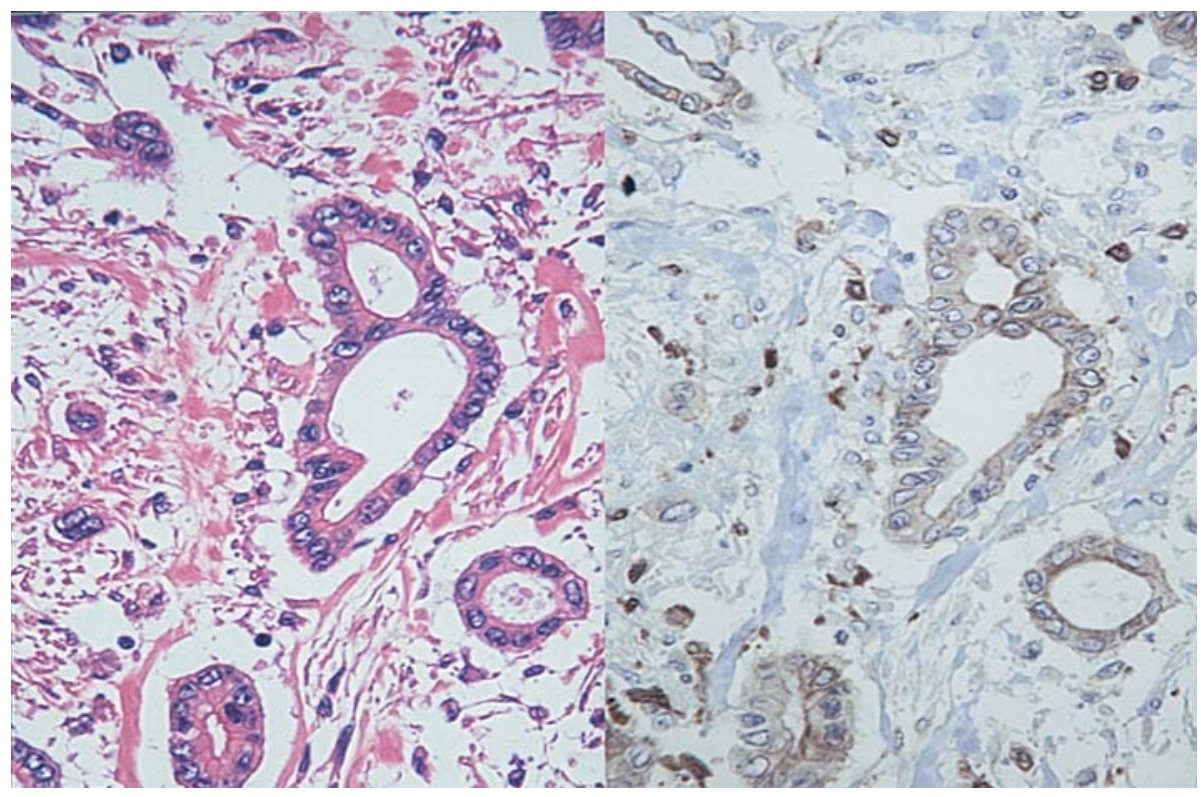

Figure 2. Immunohistochemical staining for dihydropyrimidine dehydrogenase (+++, high expression) in the primary tumor of a stage IV colorectal cancer patient with peritoneal dissemination from the long survival group (left, H\&E stain; right, DPD stain). Original magnification, x200.

The major antitumor effect of 5-FU is dependent on the inhibition of DNA synthesis by inactivation of thymidylate synthase (TS), which occurs when a ternary complex is formed between TS and methylenetetrahydrofolate $\left(\mathrm{CH}_{2} \mathrm{FH}_{4}\right)$, a metabolite of 5-FU (16-18). It has been reported that 5-FU may also be used as a chemical modulator for CPT-11, a drug that has been employed more widely in Europe and the USA than in Japan $(19,20)$. Because approximately $\geq 80 \%$ of each dose of 5-FU is metabolized by dihydropyrimidine dehydrogenase (DPD) in the liver, TS and DPD are the two key enzymes that determine the sensitivity of a tumor to therapy with 5-FU plus LV, and tumors with high TS expression/low DPD expression are considered to be most likely to respond to this combination of drugs $(17,18,21,22)$. Tumor drug sensitivity is usually assessed by molecular biological methods, such as measurement of enzyme activity in the primary tumor, immunohistochemical staining, or PCR analysis. However, there have been no reports about the TS and DPD immunopositivity of primary tumors in patients who have stage IV colorectal cancer and peritoneal dissemination without synchronous metastasis to other organs such as the liver and/or lungs. 


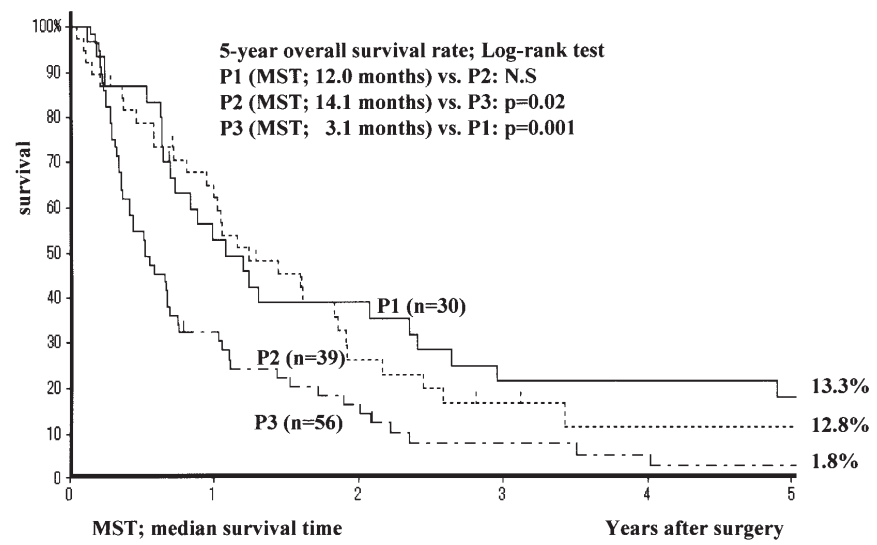

Figure 3. The 5-year overall survival (OS) rates of all 125 patients with peritoneal dissemination, including those with other synchronous metastases, were $13.3 \%$ for $\mathrm{P} 1$ patients $(\mathrm{n}=30), 12.8 \%$ for $\mathrm{P} 2$ patients $(\mathrm{n}=39)$, and $1.8 \%$ for $\mathrm{P} 3$ patients $(\mathrm{n}=56)$ ( $\mathrm{P} 1$ vs. P2, $\mathrm{p}=$ N.S.; P2 vs. P3, $\mathrm{p}=0.02$; P1 vs. P3, $\mathrm{p}=0.001$ ). The median survival time (MST) was 12.0 months for P1 patients, 14.1 months for $\mathrm{P} 2$ patients, and 3.1 months for $\mathrm{P} 3$ patients.

The objectives of the present study were to determine the 5-year overall survival (OS) rate and median survival time (MST) in stage IV colorectal cancer patients with peritoneal dissemination, and examine the 5-FU sensitivity of tumors in patients who had peritoneal dissemination without synchronous metastases to other organs such as the liver, lungs, or Virchow's node.

\section{Materials and methods}

A total of 164 patients were diagnosed as having stage IV primary colorectal cancer complicated by peritoneal dissemination during the 25-year period from 1976 to 2001 . Among these 164 patients, only 33 (20.1\%) underwent bypass and/or creation of a stoma, while 131 patients (79.9\%) underwent palliative resection of the primary tumor. Among these 131 patients, 125 had complete medical records and were followed up to assess survival. The 125 patients were divided into two groups, one with synchronous metastases to other organs such as the liver, lungs or Virchow's node $(n=61)$, and another without synchronous metastasis $(n=64)$.

Classification of peritoneal dissemination and calculation of the overall survival (OS) rate and median survival time $(M S T)$. All 125 patients enrolled in the study were divided into 3 groups according to the severity of peritoneal dissemination at the time of laparotomy. Patients in the P1 group had a few scattered metastatic nodules in the peritoneum near the primary tumor $(n=30)$, while the P2 group had a few peritoneal nodules in a distant part of the abdominal cavity $(n=39)$ (23). The P3 group had numerous nodules scattered throughout the peritoneum as far as the pouch of Douglas $(n=56)$ (23). The 5-year OS rate and MST of all 125 patients were calculated first. Then these parameters were also calculated for the 64 patients without synchronous metastases to other organs $(\mathrm{P} 1=17, \mathrm{P} 2=19$, and P3=28). The presence or absence of regional lymph node metastasis was not used as a factor for categorizing the subjects of stage IV colorectal cancer.

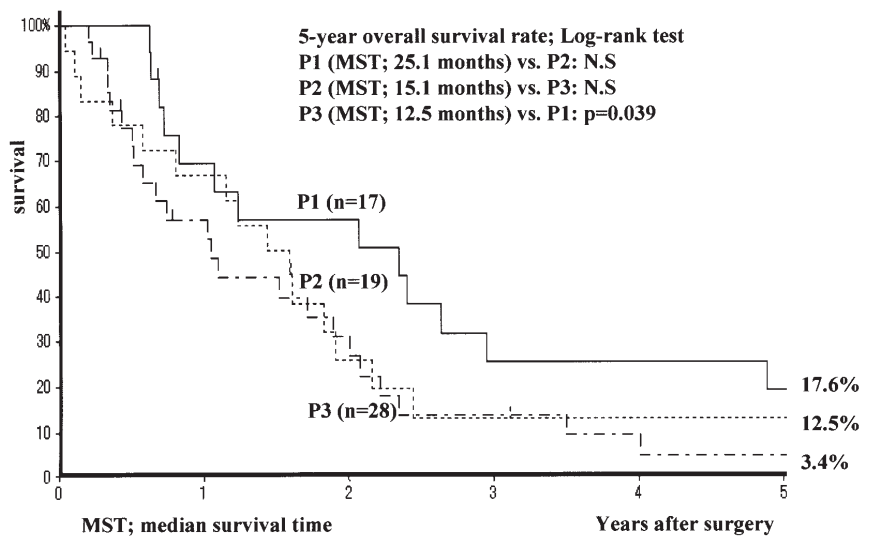

Figure 4. The 5-year overall survival (OS) rates of the 64 patients with peritoneal dissemination alone and no other synchronous metastases were $17.6 \%$ for $P 1$ patients $(n=17), 12.5 \%$ for $P 2$ patients $(n=19)$, and $3.4 \%$ for P3 patients ( $n=28)$ (P1 vs. P2, p=N.S.; P2 vs. P3, p=N.S.; P1 vs. P3, p=0.039). The median survival time (MST) was 25.1 months for P1 patients, 15.1 months for $\mathrm{P} 2$ patients, and 12.5 months for $\mathrm{P} 3$ patients.

Immunohistochemical staining for TS and DPD. The MST of 97 patients in the P1 $(n=30), P 2(n=39)$, and P3 $(n=28)$ groups was approximately 12 months. The 28 patients from the P3 group with no synchronous extraperitoneal metastases were divided into two subgroups: 1) short survival group (SSG), patients who died within 1 year; and 2) long survival group (LSG), patients who survived for $\geq 1$ year. Immunohistochemical detection of TS and DPD in the resected primary tumors was performed using a standard indirect immunoperoxidase method using polyclonal anti-TS and anti-DPD antibodies as the primary antibodies (Taiho Pharmaceutical Co., Ltd, Japan) $(17,22,24,25)$. All of the tumor cells were counted in each high-power field. Tumors without stained cells were defined as negative (-), tumors containing $<5 \%$ positive cells were classified as $(+)$, while lesions containing $5-30 \%$ positive cells were $(++)$ and those containing $\geq 30 \%$ positive cells were $(+++)$. Either $(-)$ or $(+)$ was defined as a low level of expression, while $(++)$ or $(+++)$ was categorized as a high level of expression (Figs. 1 and 2) (26-28). Patients with high TS and low DPD expression in their tumors were defined as being more likely to respond to $5 \mathrm{FU}+\mathrm{LV}$ therapy.

Statistical analysis. To assess the 5-year OS of each subgroup, survival rates were calculated using the Kaplan-Meier method, and the log-rank test was used for comparison between the P1, P2, and P3 groups. The $\chi^{2}$ test was also used to examine the significance of differences between the SSG and LSG. A $\mathrm{P}$-value of $<0.05$ was considered to indicate a significant difference in all analyses.

\section{Results}

In all subjects, including those with synchronous metastases to other organs such as the liver, lungs, or Virchow's node, the 5-year OS rate was $13.3 \%$ for P1 patients $(n=30), 12.8 \%$ for $\mathrm{P} 2$ patients $(\mathrm{n}=39)$, and $1.8 \%$ for $\mathrm{P} 3$ patients $(\mathrm{n}=56)(\mathrm{P} 1$ vs. P2, p=N.S.; P2 vs. P3, p=0.02; P1 vs. P3, p=0.001) (Fig. 3), while the MST was 12.0 months for $\mathrm{P} 1$ patients, 14.1 months 
Table I. Expression of thymidilate synthase (TS) and dihydropyrimidine dehydrogenase (DPD) in the primary tumors of 28 patients with stage IV colorectal cancer and P3 peritoneal dissemination.

\begin{tabular}{|c|c|c|c|c|c|}
\hline \multirow[b]{2}{*}{ Total $(\mathrm{n}=28)$} & \multicolumn{2}{|c|}{ TS } & \multicolumn{2}{|c|}{ DPD } & \multirow{2}{*}{$\frac{\text { TS \& DPD }}{\text { High-TS/low-DPD }}$} \\
\hline & High-level & Low-level & High-level & Low-level & \\
\hline $\operatorname{SSG}(n=13)$ & $7.7 \%(1 / 13)$ & $92.3 \%(12 / 13)$ & $38.5 \%(5 / 13)$ & $61.5 \%(8 / 13)$ & $7.7 \%(1 / 13)^{\mathrm{a}}$ \\
\hline LSG (n=15) & $80.0 \%(12 / 15)$ & $20.0 \%(3 / 15)$ & $33.3 \%(5 / 15)$ & $66.7 \%(10 / 15)$ & $46.7 \%(7 / 15)$ \\
\hline
\end{tabular}

The patients were divided into a short survival group ( $\mathrm{SSG}, \mathrm{n}=13$ ) and long survival group (LSG, $\mathrm{n}=15$ ). TS and DPD expression was classified as low $(-,+)$ or high $(++,+++)$ with immunohistochemical staining. TS, thymidylate synthase; DPD, dihydropyrimidine dehydrogenase. ${ }^{\mathrm{a}} \mathrm{p}=0.028$.

for $\mathrm{P} 2$ patients, and 3.1 months for $\mathrm{P} 3$ patients (Fig. 3). Among the subjects without synchronous metastases to other organs, the 5-year OS rate was $17.6 \%$ for $\mathrm{P} 1$ patients $(\mathrm{n}=17), 12.5 \%$ for $\mathrm{P} 2$ patients $(\mathrm{n}=19)$, and $3.4 \%$ for $\mathrm{P} 3$ patients $(\mathrm{n}=28)(\mathrm{P} 1$ vs. P2, p=N.S.; P2 vs. P3, p=N.S.; P1 vs. P3, p=0.039) (Fig. 4), while the MST was 25.1 months for P1 patients, 15.1 months for $\mathrm{P} 2$ patients, and 12.5 months for $\mathrm{P} 3$ patients (Fig. 4).

Among 28 patients from the P3 group who only had peritoneal dissemination, 13 belonged to the SSG (46.4\%) and 15 to the LSG (53.6\%). In the SSG, tumor expression of TS was high in $7.7 \%$ (1/13) and low in $92.3 \%$ (12/13), while tumor expression of DPD was high in $38.5 \%$ (5/13) and low in $61.5 \%(8 / 13)$ (Table I). In the LSG, TS expression was high in $80.0 \%(12 / 15)$ and low in $20.0 \%(3 / 15)$, while DPD expression was high in $33.3 \%$ (5/15) and low in $66.7 \%(10 / 15)$ (Table I). The percentage of patients with high TS/low DPD expression in their tumors was only $7.7 \%(1 / 13)$ in the SSG versus $46.7 \%$ (7/15) in the LSG ( $\mathrm{p}=0.028)$ (Table I).

\section{Discussion}

The prognosis of advanced colorectal cancer complicated by peritoneal dissemination is extremely poor. The mechanism of metastasis/recurrence and the biological behavior of the tumor differ between colorectal cancer and gastric cancer. Even in patients with recurrence and/or metastasis of colorectal cancer to organs such as the liver or lungs, volume reduction surgery may be performed if the metastasis is a solitary nodule or 2 to 3 resectable masses, and a relatively good prognosis can be expected, unlike with gastric cancer $(3,4,26-28)$. In the present study, analysis of the factor with the most significant influence on survival revealed that patients with Virchow's node metastasis had the worst prognosis among those with synchronous metastases to other organs $(n=61$, data not shown). It is often difficult to perform comparative analyses involving multiple factors/variables in patients with advanced cancer because of their diverse clinical background and history of various therapies. Accordingly, we performed an analysis of the factors determining survival/prognosis in patients who only had peritoneal dissemination without synchronous metastasis to other organs. We found that the survival of P1 patients, in whom peritoneal involvement was limited to near the primary tumor, was better than that of $\mathrm{P} 3$ patients with widespread dissemination $(\mathrm{p}=0.039)$. In the $\mathrm{P} 1$ group, $52.9 \%$ (9/17) of patients underwent complete resection of their peritoneal nodules in addition to resection of the primary tumor, and the results indicated that volume reduction surgery should be considered for P1 patients. Even in P3 patients with dissemination of small tumor nodules throughout the peritoneum, preoperative diagnosis of peritoneal involvement is difficult if they have no obstruction or ascites. In these patients, the metastases cannot be removed completely, so a bypass or stoma is created to prevent small bowel obstruction because it is considered that resection of the primary tumor will not improve survival. In the present study, 20.1\% (33/164) of patients did not undergo palliative resection of the primary tumor and were scheduled to receive chemotherapy. Because of their poor general condition due to worsening cachexia, however, $63.6 \%$ of these patients (21/33) either received no chemotherapy or withdrew from palliative chemotherapy at an early stage of treatment.

The most significant determinant of response to chemotherapy is the sensitivity of residual cancer cells and disseminated tumor nodules, particularly intraperitoneal lesions that can cause small bowel obstruction. Therefore, it may be more useful to perform direct examination of the chemosensitivity of tumor nodules rather than measuring TS and DPD activity in homogenates of the primary tumor or performing immunohistochemical staining of the primary. It has been reported that relatively high expression of both TS and DPD is found in neoplastic tissue, while little or no DPD expression is found in non-neoplastic tissue $(17,22)$. However, none of the previous studies included a detailed examination of pathological changes at sites other than the primary tumor, such as peritoneal nodules. In this study, among 125 patients who underwent resection of the primary tumor, peritoneal nodules were only collected from 19 patients (15.2\%). Immunohistochemical examination of TS and DPD expression was performed in 7 of these 19 patients (36.8\%) but preliminary examination of a few small metastatic nodules showed that staining for TS and DPD was weak and unstable. Accordingly, we decided to examine 5-FU sensitivity by immunostaining of the primary tumors instead. This finding suggests that fresh peritoneal tumor nodules should be collected during surgery for the assessment of sensitivity to anticancer agents.

Among 13 patients in the SSG, 10 (76.9\%) underwent postoperative treatment with a 5-FU derivative, while the other $3(23.1 \%)$ were untreated. Of the 10 patients treated with a 5-FU derivative, $5(50.0 \%)$ received an oral fluoropyrimidine alone, while the other $5(50.0 \%)$ were given 
combination therapy with intravenous 5-FU and LV. None of these patients had tumors with high TS/low DPD expression, which indicates higher sensitivity to 5-FU, and treatment with 5-FU was not found to be very effective in this group. Combination chemotherapy with other drugs, such as CPT-11 and/or L-OHP, which has been more widely used in Western countries than in Japan, may be needed to improve the limited efficacy of 5-FU monotherapy $(8,19,20)$. Among 15 patients in the LSG, $9(60.0 \%)$ underwent postoperative treatment with a 5-FU derivative, while the other $6(40.0 \%)$ were untreated. Of the 9 patients treated with 5-FU derivatives, $2(22.2 \%)$ received an oral fluoropyrimidine alone, while the other $7(77.8 \%)$ were given intravenous 5-FU plus LV. Of the 7 patients receiving combination therapy, $5(71.4 \%)$ had tumors that showed high TS/low DPD expression, indicating that they were more likely to respond to the combination of 5-FU and LV.

To prevent postoperative impairment of the quality of life (QOL), oral anticancer agents are increasingly prescribed as palliative therapy after early discharge of stage IV colorectal cancer without perioperative complications (6-8). Oral anticancer therapy has a long history in Japan and various 5-FU derivatives have been used since the early 1980s. Some studies have even suggested that oral anticancer therapy can be pharmacologically and clinically equivalent to intravenous therapy. In Europe and the USA, oral anticancer therapy has attracted considerable attention since the 1999 Conference of the American Society of Clinical Oncology in New Orleans, where the efficacy of oral agents was reported (Pazdur R, et al, Proc ASCO 18: abs. 1009, 1999; Carmichael J, et al, Proc ASCO 18: abs. 1015, 1999). Similar to combination therapy with intravenous 5-FU and LV, which is the gold standard for the treatment of colorectal cancer, new regimens that may further improve the efficacy of oral fluoropyrimidines, such as chemical modulation by oral LV and use of a DPDinhibiting fluoropyrimidine (TS-1, tegafur/gimeracil/oteracil potassium; Taiho Pharmaceutical Co., Ltd., Tokyo, Japan), have been reported $(7,29,30)$. It has been suggested that improvement of the survival rate provides the only true benefit for patients with advanced or recurrent cancer $(31,32)$. In patients with stage IV colorectal cancer complicated by peritoneal dissemination, however, balanced tumor dormancy therapy should be given to extend their survival by reducing the tumor burden, while maintaining a better QOL. It is important to choose the most appropriate therapy for each patient during the perioperative period to avoid problems related to resistance to 5-FU derivatives.

\section{Acknowledgements}

This study was supported by grants from the Occult Neoplastic Cells Research and Study Group (\#2005-2291; Tokai University Hachioji Hospital, Hachioji, Tokyo, Japan) and the Research and Study Program of Tokai University Educational System General Research Organization (\#2005-31; Tokai University Hospital, Isehara, Kanagawa, Japan).

\section{References}

1. Journal of Health and Welfare Statistics. Health and Welfare Statistics Association, Tokyo 51: pp46-49, 2004.
2. Multi-Institutional Registry of Large Bowel Cancer in Japan: Cases treated in 1994. Vol. 23. Japanese Society for Cancer of the Colon and Rectum, Tokyo, 2002.

3. Galandiuk S, Wieand HS, Moertel CG, Cha SS, Fitzgibbons RJ, Pemberton JH and Wolff BG: Pattern of recurrence after curative resection of carcinoma of the colon and rectum. Surg Gynecol Obst 174: 27-32, 1992.

4. Ambiru S, Miyazaki M, Ito H, Nakagawa K, Shimizu H, Kato A, Nakamura S, Omoto $\mathrm{H}$ and Nakajima N: Resection of hepatic and pulmonary metastases in patients with colorectal carcinoma. Cancer 82: 274-278, 1998.

5. Mukai M, Hinoki T, Tajima T, Sato S, Nakasaki H, Ogoshi K and Makuuchi H: Complete remission after combination chemotherapy for stage IV gastric cancer with peritoneal dissemination and liver matastases; case report. Ann Cancer Res Therap 11: 169-181, 2003.

6. Mukai M, Tokunaga N, Yasuda S, Mukohyama S, Kameya T, Ishikawa K, Iwase H, Suzuki T, Ishida H, Sadahiro S and Makuuchi H: Long-term survival after immunochemotherapy for juvenile colon cancer with peritoneal dissemination: a case report. Oncol Rep 7: 1343-1347, 2000.

7. Mukai M, Moriya H, Himeno S, Oida Y, Mukohyama S, Nishi T, Nakasaki H, Satoh S and Makuuchi H: Efficacy of oral UFT plus leucovorin therapy for colon cancer with ovarian and multiple liver metastases: report of two cases. Oncol Rep 8: 1079-1083, 2001

8. Mukai M, Oida Y, Mukoyama S, Okamoto Y, Ito I, Nakasaki H, Kawai K, Sato S and Makuuchi H: Efficacy of combination chemotherapy for stage IV colon cancer with extensive peritoneal dissemination and multiple liver metastases: a case report. Oncol Rep 9: 1339-1343, 2002.

9. Hasegawa K, Maruyama M, Maruyama S, Tamura N, Miyawaki Y, Yakashima I, Koide A, Ebuchi M and Sakoma T: Evaluation of peritoneal cytology in colorectal carcinoma. Jpn J Cancer Chemother 32: 1701-1706, 2005.

10. Bree ED, Witkamp AJ and Zoetmulder FAN: Intraperitoneal chemotherapy for colorectal cancer. J Surg Oncol 79: 46-61, 2002.

11. Sugarbeker PH: Surgical management of carcinomatosis from colorectal cancer. Clin Colon Rectal Surg 18: 190-203, 2005.

12. Imada $T$ and Mastumoto A: Mechanism by which peritoneal disseminated metastasis develops in gastric cancer. Nippon Geka Gakkai Zassi 87: 593-603, 1986 (in Japanese).

13. Petrelli N, Douglass Jr HO, Herrera L, Russell D, Stablein DM, Bruckner HW, Mayer RJ, Schinella R, Green MD, Muggia FM, Megibow A, Greenwald ES, Bukowski RM, Harris J, Levin B, Gaynor E, Loutfi A, Kalser MH, Barkin JS, Benedetto P, Woolley PV, Nauta R, Weaver DW and Leichman LP for the Gastrointestinal Tumor Study Group: The moduration of fluorouracil with leucovorin in metastatic colorectal carcinoma: A prospective randomized phase III trial. J Clin Oncol 7: 1419-1426, 1989.

14. Poon MA, O'Connell MJ, Wieand HS, Krook JE, Gerstner JB, Tschetter LK, Levitt R, Kardinal CG and Mailliard JA: Biochemical modulation of fluorouracil with leucovorin: confirmatory evidence of improved therapeutic efficacy in advanced colorectal cancer. J Clin Oncol 9: 1967-1972, 1991

15. De Gramont A, Bosset J-F, Milan C, Rougier P, Bouché O, Etienne P-L, Morvan F, Louvet C, Guillot T, François E and Bedenne L: Randomized trial comparing monthly low dose leucovorin and fluorouracil bolus with bimonthly high-dose leucovorin and fluorouracil bolus plus continuous infusion for advanced colorectal cancer. J Clin Oncol 15: 808-815, 1997.

16. Berger SH and Hakala MT: Relationship of dUMP and free FdUMP pools to inhibition of thymidylate synthase by 5fluorouracil. Mol Pharmacol 25: 303-309, 1984.

17. Okabe H, Tsujimoto $\mathrm{H}$ and Fukushima M: Preparation of the antibodies against recombinant human thymidylate synthase for the detection of its intratumoral levels and application to sensitivity-study of 5-fluorouracil. Oncol Rep 4: 685-690, 1997.

18. Takenoue T, Nagawa H, Matsuda K, Fujii S, Nita ME, Kitayama J, Tsuruo T and Muto T: Relation between thymidylate synthase expression and survival in colon carcinoma, and determination of appropriate application of 5-fluorouracil by immunohistochemical method. Ann Surg Oncol 7: 193-198, 2000.

19. Saltz LB, Cox JV, Blanke C, Rosen LS, Fehrenbacher L, Moore MJ, Maroun JA, Ackland SP, Locker PK, Pirotta N, Elfring GL and Miller LL for the Irinotecan Study Group: Irinotecan plus fluorouracil and leucovorin for metastatic colorectal cancer. N Engl J Med 343: 905-914, 2000. 
20. Douillard JY, Cunningham D, Roth AD, Navarro M, James RD, Karasek P, Jandic P, Iveson T, Carmichael J, Alaki M, Gruia G, Awad L and Rougier P: Irinotecan combined with fluorouracil compared with fluorouracil alone as first-line treatment for metastatic colorectal cancer: a multicentre randomized trial. Lancet 355: 1041-1047, 2000.

21. Takenoue T, Kitayama J, Takei Y, Umetani N, Matsuda K, Nita ME, Hatano K, Tsuruo T and Nagawa H: Characterization of dihydropyrimidine dehydrogenase on immunohistochemistry in colon carcinoma, and correlation between immunohistochemical score and protein level or messenger RNA expression. Ann Oncol 11: 273-279, 2000.

22. Kamoshida S, Shiogama K, Matsuoka H, Matsuyama A, Shimomura R, Inada K, Maruta M and Tsutsumi Y: Immunohistochemical demonstration of dihydropyrimidine dehydrogenase in normal and cancerous tissues. Acta Histochem Cytochem 36: 471-479, 2003.

23. Japanese Society for Cancer of the Colon and Rectum: General Rules for Clinical and Pathological Studies on Cancer of the Colon, Rectum and Anus. 6th edition. Kanehara and Co. Ltd., Tokyo, 1998.

24. Nakane PK and Pierce GB: Enzyme-labeled antibodies: preparation and application for localization of antigens. $J$ Histochem Cytochem 14: 929-931, 1966.

25. Nakane PK and Pierce GB: Enzyme-labeled antibody for the light and electron microscopic localization of tissue antigens. J Cell Biol 33: 307-318, 1967.

26. Mukai M, Sato S, Ninomiya H, Wakui K, Komatsu N, Tsuchiya K, Nakasaki $\mathrm{H}$ and Makuuchi $\mathrm{H}$ : Recurrence and 5-FU sensitivity of stage III/Dukes' C colorectal cancer with occult neoplastic cells in lymph node sinuses. Oncol Rep 14: 1165-1169, 2005.
27. Mukai M, Sato S, Ninomiya H, Wakui K, Komatsu N, Tsuchiya K, Nakasaki H and Makuuchi H: Recurrence and 5-FU sensitivity of stage II/Dukes' B colorectal cancer with occult neoplastic cells in lymph node sinuses. Oncol Rep 14: 1171-1176, 2005.

28. Mukai M, Sato S, Ninomiya H, Wakui K, Komatsu N, Tsuchiya K, Tajima T, Nakasaki $\mathrm{H}$ and Makuuchi $\mathrm{H}$ : Recurrence and 5-FU sensitivity of stage II/III gastric cancer with occult neoplastic cells in lymph node sinuses. Oncol Rep 14: 1501-1505, 2005.

29. Koizumi W, Kurihara M, Nakano S and Hasagawa K for the S-1 Cooperative Gastric Cancer Study Group: Phase II study of S-1, a novel oral derivative of 5-fluorouracil, in advanced gastric cancer. Oncology 58; 191-197, 2000.

30. Sugimachi K, Maehara Y, Horikoshi N, Shimada Y, Sakata Y, Mitachi Y, Taguchi T and the S-1 Gastrointestinal Cancer Study Group: An early phase II study of oral S-1, a newly developed 5 -fluorouracil derivative for advanced and recurrent gastrointestinal cancers. Oncology 57: 202-210, 1999.

31. Feinstein AR, Sosin DM and Wells CK: The Will Rogers phenomenon: Stage migration and new techniques as a source of misleading statistics for survival in cancer. N Engl J Med 312: 1604-1608, 1985.

32. Bunt AMG, Hermans J, Smit VTHBM, van de Velde CJH, Fleuren GL and Bruijn JA: Surgical/pathologic stage migration confounds comparisons of gastric cancer survival rates between Japan and Western countries. J Clin Oncol 13: 19-25, 1995. 